\title{
A tear fluid proteome of Parkinson's disease
}

Henrik Zetterberg ${ }^{1,2,3,4}$ \& Yaroslau Compta ${ }^{5,6,7}$

\author{
${ }^{1}$ Department of Psychiatry and Neurochemistry, Institute of Neuroscience and Physiology, the Sahlgrenska Academy at the University of \\ Gothenburg, Mölndal, Sweden \\ ${ }^{2}$ Clinical Neurochemistry Laboratory, Sahlgrenska University Hospital, Mölndal, Sweden \\ ${ }^{3}$ Department of Neurodegenerative Disease, UCL Institute of Neurology, Queen Square, London, UK \\ ${ }^{4} U K$ Dementia Research Institute at UCL, London, UK \\ ${ }^{5}$ Parkinson's disease \& Movement Disorders Unit, Neurology Service, Hospital Clínic de Barcelona, Barcelona, Catalonia, Spain \\ ${ }^{6}$ Parkinson's disease and other degenerative movement disorders lab, IDIBAPS, Barcelona, Catalonia, Spain \\ ${ }^{7}$ Institut Clínic de Neurociències, Universitat de Barcelona, Barcelona, Catalonia, Spain
}

Given the inaccessibility of the brain, attention has turned to body fluids, such as plasma and cerebrospinal fluid, as potential sources of diagnostic and prognostic biomarkers for neurodegenerative disease. This approach has proven successful in Alzheimer's disease (AD), but it has been much harder to find reliable fluid biomarkers for Parkinson's disease (PD) [1]. The concentration of Lewy body pathology-associated $\alpha$-synuclein in cerebrospinal fluid (CSF) is reduced in PD patients, but with a large overlap between patient and control groups [2], and fluid biomarkers for neurodegeneration, such as neurofilament light (NfL), are mostly negative, at least in early stages of the disease [3]. In the current issue of Parkinsonism \& Related Disorders, Boerger and colleagues report on a novel approach to identify PD biomarkers: proteomic analysis of tear fluid [4].

What is the potential relationship between tear fluid composition and PD neuropathology? Human tear fluid is a complex biological mixture containing high concentrations of proteins, including proteins of relevance to PD pathogenesis, e.g., $\alpha$-synuclein [5]. Whether $\alpha$-synuclein-containing Lewy bodies can be detected in the lacrimal gland is, to the best of our knowledge, unknown, but it is clear that the pathology is not limited to the brain but extends to peripheral tissues, including gastrointestinal tract, salivary glands, olfactory mucosa, skin, retina, adrenal gland, and heart [6]. Moreover, regardless of its source (peripheral blood, CSF, or other fluids such as in this case tear fluid), directly measuring $\alpha$-synuclein might theoretically fail to distinguish among different synucleinopathies, which besides PD include other conditions often difficult to be differentiated from PD, such as multiple system atrophy (MSA) or dementia with Lewy bodies (DLB). Hence, approaches that explore proteins other than $\alpha$-synuclein could be of potential interest to discriminate PD not only from healthy controls or other non-synucleinopathy conditions (tauopathies such as progressive supranuclear palsy [PSP] and corticobasal degeneration [CBD]), but also from other closely related synucleinopathies. In this vein, another potential link between the brain and tear fluid composition concerns the innervation of the lacrimal glands by the trigeminal V1 (fifth cranial) nerve; tear fluid secretion by lacrimal glands is stimulated by cholinergic neurons. The production, packaging and secretion of specific proteins into tears may be regulated by changes in nerve function to lacrimal glands. Analysis of any alteration in the secretion of proteins into tears may thus reveal biomarkers of potential relevance to PD.

Boerger et al. collected tear fluid samples from 36 PD patients and 18 neurologically healthy control individuals, using Schirmer tear test strips from which proteins where extracted, digested and subjected to mass spectrometric analysis [4]. A total of 571 proteins could be identified, 31 of which 
were exclusively detected in the PD group. Twenty-one proteins were increased in PD patients compared with controls, whereas 19 proteins were significantly decreased. When classifying the differentially expressed proteins based on function, they were predominantly involved in immune response and lipid metabolism.

This study is not without caveats. Besides the inclusion on of a small group of neurodegenerative diseases other than PD, there is no proper comparison of PD to other parkinsonisms, either secondary (drug-induced, vascular) or degenerative (be it synucleinopathies such as MSA and DLB, be it tauopathies like PSP and CBD). Therefore, it remains unknown whether the observed differences are disease-specific, or not. Additionally, the control group was smaller than the PD one, and thus larger samples with more stringent correction for multiplicity are warranted to further explore the preliminary findings from this pilot study. Finally, protein intensities were measured corresponding to the pooled samples, but not individual patient samples, so the direct discriminant value of these proteomic findings also remains to be further explored at the individual level.

All this notwithstanding, the findings by Boerger et al [4] are promising in their own right and moreover have the asset of being derived from a very accessible biofluid. This now calls for further replication studies on larger patient cohorts, examining different stages of PD, as well as differential diagnostics aspects of the biomarker candidates with other neurodegenerative parkinsonisms (both synucleinopathies and taoupathies) as well as other neurodegenerative conditions.

\section{Conflicts of interest:}

$\mathrm{HZ}$ has served at scientific advisory boards for Roche Diagnostics, Samumed, Wave and CogRx, has given lectures in symposia sponsored by Biogen and Alzecure, and is a co-founder of Brain Biomarker Solutions in Gothenburg AB, a GU Ventures-based platform company at the University of Gothenburg (all outside submitted work).

\section{REFERENCES}

[1] Lleó A, Cavedo E, Parnetti L, Vanderstichele H, Herukka SK, Andreasen N, Ghidoni R, Lewczuk P, Jeromin A, Winblad B, Tsolaki M, Mroczko B, Visser PJ, Santana I, Svenningsson P, Blennow $\mathrm{K}$, Aarsland D, Molinuevo JL, Zetterberg H, Mollenhauer B. Cerebrospinal fluid biomarkers in trials for Alzheimer and Parkinson diseases. Nat Rev Neurol. 2015 Jan;11(1):41-55. PMID: 25511894

[2] Mollenhauer B, Locascio JJ, Schulz-Schaeffer W, Sixel-Döring F, Trenkwalder C, Schlossmacher MG. $\alpha$-Synuclein and tau concentrations in cerebrospinal fluid of patients presenting with parkinsonism: a cohort study. Lancet Neurol. 2011 Mar;10(3):230-40. PMID: 21317042.

[3] Khalil M, Teunissen CE, Otto M, Piehl F, Sormani MP, Gattringer T, Barro C, Kappos L, Comabella M, Fazekas F, Petzold A, Blennow K, Zetterberg H, Kuhle J. Neurofilaments as biomarkers in neurological disorders. Nat Rev Neurol. 2018 Oct;14(10):577-589. PMID: 30171200.

[4] Boerger M, Funke S, Leha A, Roser AE, Wuestemann AK, Maass F, Bähr M, Grus F, Lingor P. Proteomic analysis of tear fluid reveals disease-specific patterns in patients with Parkinson's disease - A pilot study. Parkinsonism Relat Disord. 2019 Mar 6. doi: 10.1016/j.parkreldis.2019.03.001. PMID: 0876839. 
[5] Hamm-Alvarez SF, Okamoto CT, Janga SR, Feigenbaum D, Edman MC, Freire D, Shah M, Ghanshani R, Mack WJ, Lew MF. Oligomeric $\alpha$-synuclein is increased in basal tears of Parkinson's patients. Biomark Med. 2019 Jul 2. doi: 10.2217/bmm-2019-0167. PMID: 31262201.

[6] Ma LY, Liu GL, Wang DX, Zhang MM, Kou WY, Feng T. Alpha-Synuclein in Peripheral Tissues in Parkinson's Disease. ACS Chem Neurosci. 2019 Feb 20;10(2):812-823. PMID: 30714719. 